Revue d'histoire de l'Amérique française

QV REVUE D.HISTOIRE DE L'AMÉRIQUE FRANÇAISE

\title{
La publicité américaine à la radio canadienne : le cas du réseau français de Radio-Canada, 1938-1958
}

\section{Michel Filion}

Volume 51, numéro 1, été 1997

URI : https://id.erudit.org/iderudit/305623ar

DOI : https://doi.org/10.7202/305623ar

Aller au sommaire du numéro

\section{Éditeur(s)}

Institut d'histoire de l'Amérique française

\section{ISSN}

0035-2357 (imprimé)

1492-1383 (numérique)

Découvrir la revue

\section{Citer cet article}

Filion, M. (1997). La publicité américaine à la radio canadienne : le cas du réseau français de Radio-Canada, 1938-1958. Revue d'histoire de l'Amérique française, 51(1), 71-92. https://doi.org/10.7202/305623ar
Résumé de l'article

La création de Radio-Canada en 1936 visait à prémunir la radio naissante de l'influence américaine, dans sa structure et dans son contenu, et à favoriser ainsi l'éclosion de l'identité canadienne. En dépit de ce mandat d'ordre éducatif, la société d'État dut se tourner vers la publicité afin d'assumer les coûts que l'expansion du réseau national réclamait. Fondée sur le dépouillement d'un ensemble documentaire jusque-là inexploré, les fiches de commanditaires de la Société Radio-Canada, notre recherche démontre que la commandite d'émissions est massivement venue de firmes américaines. On a pu craindre, avec raison, que cette commercialisation des ondes n'injecte dans le tissu culturel canadien des produits étrangers sous forme de programmes. Mais, au contraire de ce qui s'est produit au Canada anglais, la publicité a davantage ancré la radio de Radio-Canada dans le Québec francophone en encourageant la production locale d'émissions de divertissement populaire. 


\title{
LA PUBLICITÉ AMÉRICAINE À LA RADIO CANADIENNE: LE CAS DU RÉSEAU FRANÇAIS DE RADIO-CANADA,1938-1958
}

\author{
MICHEL FILION \\ Département des sciences de l'éducation \\ Université du Québec à Hull
}

\section{RÉSUMÉ}

La création de Radio-Canada en 1936 visait à prémunir la radio naissante de l'influence américaine, dans sa structure et dans son contenu, et à favoriser ainsi l'éclosion de l'identité canadienne. En dépit de ce mandat d'ordre éducatif, la société d'État dut se tourner vers la publicité afin d'assumer les coûts que l'expansion du réseau national réclamait. Fondée sur le dépouillement d'un ensemble documentaire jusque-là inexploré, les fiches de commanditaires de la Société Radio-Canada, notre recherche démontre que la commandite d'émissions est massivement venue de firmes américaines. On a pu craindre, avec raison, que cette commercialisation des ondes n'injecte dans le tissu culturel canadien des produits étrangers sous forme de programmes. Mais, au contraire de ce qui s'est produit au Canada anglais, la publicité a davantage ancré la radio de Radio-Canada dans le Québec francophone en encourageant la production locale d'émissions de divertissement populaire.

\begin{abstract}
The Canadian Broadcasting Corporation was created in 1936 to foster nationwide identity by the means of a distinctive Canadian radio structure and content devoted to education rather than commercial profits as in the United States. The development of a national network nevertheless called for revenues other than governmental funding. Based on a set of records until now unexplored, the CBC sponsors card index, this research shows that program sponsorship massively came from American firms and brought American programs accross the border. But, in francophone Québec, this American sponsorship led to the production of local popular entertainment programs which made the Canadian Broadcasting Corporation's French network deeper integrated in its cultural environment.
\end{abstract}




\section{INTRODUCTION}

La pénétration américaine au Canada sous forme de programmes et de publicité est une préoccupation depuis longtemps répandue. Dès 1929, le rapport de la Commission royale d'enquête sur la radiodiffusion au Canada (commission Aird) réclamait l'intervention du gouvernement fédéral - notamment en matière de financement de la radio - pour contrer le jeu des forces commerciales préjudiciables à l'éclosion et à la sauvegarde de l'identité canadienne ${ }^{1}$. Ce problème conserve toute son actualité, en particulier depuis le désengagement progressif de l'État ${ }^{2}$.

Or, à l'instar de la radio privée, celle de la Société Radio-Canada fut soumise aux impératifs du commerce, car elle fut partiellement financée par la réclame dès sa création en 1936. Fut-elle ainsi un agent de l'influence étrangère, bien que sa raison d'être visait justement à prémunir le Canada du système américain entièrement voué au profit commercial? À l'aide d'un corpus documentaire encore inexploité, nous avons voulu explorer plus avant la question de la publicité diffusée sur les ondes de Radio-Canada au cours de ses deux premières décennies, c'est-à-dire depuis la création d'un réseau français autonome jusqu'à ce que la loi de 1958 modifie substantiellement le paysage de la radiodiffusion canadienne.

\section{I - LE PROBLÈME HISTORIQUE DE LA PUBLICITÉ À LA RADIO}

L'histoire de la publicité dans les médias électroniques canadiens demeure méconnue, en dépit du nombre d'études sur la radiodiffusion consacrées pour la plupart à son économie politique ou plus particulièrement à la réclame télévisuelle ${ }^{3}$. Certains ouvrages méritent néanmoins d'être signalés, comme celui de O. J. Firestone dont la perspective est strictement économique et qui, malgré le recours à quelques séries chiffrées remontant à 1946, reste plutôt un exercice de prospec-

1. J. Aird, Rapport de la Commission royale d' enquête sur la radiodiffusion au Canada (Ottawa, Imprimeur du Roi, 1929).

2. Le rapport Juneau faisait encore récemment le parallèle entre la radio commerciale et la pénétration américaine, en soulignant les avantages de la quasi-disparition de la radio publique depuis le milieu des années 1970: «La radio de la SRC reflète les principes qui, selon nous, devraient régir la SRC et la radiodiffusion publique. Ses émissions sont entièrement différentes de celles qu'offrent les radiodiffuseurs privés. Elles sont informatives, intelligentes, variées, culturelles et presque exclusivement canadiennes. Elles ont de solides racines dans les régions. Elles sont dégagées des contraintes de la publicité.» P. Juneau, Faire entendre nos voix. Rapport $d u$ Comité d'examen des mandats SRC, ONF, Téléfilm (Ottawa, Ministère des Approvisionnements et Services, 1996), 49.

3. P. Rutherford, The New Icons? The Art of Television Advertising (Toronto, University of Toronto Press, 1994). 
tive ${ }^{4}$. Benjamin Singer accorde une soixantaine de pages au développement de la publicité en Amérique du Nord: bien que son explication escamote la situation canadienne, elle demeure intéressante quant au rôle normatif joué par la réclame publicitaire au fil du temps et suggère que cette dernière permet de sonder un aspect considérable de la culture de masse ${ }^{5}$. Dans leur histoire de la publicité au Canada, H. E. Stephenson et $\mathrm{C}$. McNaught ont consacré un chapitre à la radio dans lequel ils mentionnent que les firmes américaines, souvent par filiales interposées, investissent rapidement dans la radio de la Canadian Broadcasting Corporation et participent à l'agencement de sa programmation en commanditant des émissions populaires, d'origine américaine, disponibles à prix avantageux ${ }^{6}$. Dans un manuel consacré à la vente et à la publicité, Philippe Girardet et Paul Legendre soulignaient en 1951 la mainmise des commanditaires et des agences anglo-saxonnes sur la conception des campagnes publicitaires qui, traduites plutôt qu'adaptées, étaient destinées au Québec ${ }^{7}$. Plus récemment, Frederick Elkin a montré qu'à la veille de la Révolution tranquille, les grandes firmes commencent à reconnaître le poids de la culture - et de l'identité nationale - dans la mise en œuvre de campagnes publicitaires efficaces ${ }^{8}$. C'est à ce moment que les Québécois francophones s'approprient leur publicité, jusque-là calquée sur celle destinée aux marchés canadien-anglais et américain. La fondation du Publicité-club en 1959 constitue à cet égard un jalon important que souligne le livre de Jean-Marie Allard'.

Par ailleurs, les historiens de la radiodiffusion font souvent référence au tumultueux débat qui a entouré l'entrée de la publicité dans l'établissement et le développement d'une radio «nationale» au $\mathrm{Ca}$ nada ${ }^{10}$. Après que le gouvernement fédéral eut décidé d'intervenir à la fin des années 1920, souvent au grand dam de ses homologues provinciaux et des radiodiffuseurs privés, la commandite fut en effet l'objet de discorde par excellence entre ceux, politiciens et autres artisans de

4. O. J. Firestone, Broadcast Advertising in Canada: Past and Future Growth (Ottawa, Presses de l'Université d'Ottawa, 1966).

5. B. Singer, Advertising \& Society (Don Mills, Addison-Wesley Publishers, 1986).

6. H. E. Stephenson et C. McNaught, The Story of Advertising in Canada. A Chronicle of Fifty Years (Toronto, The Ryerson Press, 1940).

7. P. Girardet et P. Legendre, La vente et la publicité (Montréal, Bélisle, 1951).

8. F. Elkin, Rebels and Colleagues. Advertising and Social Changes in French Canada (Montréal, McGill-Queen's University Press, 1973). 1989).

9. J.-M. Allard, La pub: 30 ans de publicité au Québec (Montréal, Libre expression,

10. A. Weir, The Struggle for National Broadcasting in Canada (Toronto, McClelland and Stewart, 1965); F. Peers, The Politics of Canadian Broadcasting, 1920-1951 (Toronto, University of Toronto Press, 1969); M. Raboy, Missed Opportunities: the Story of Canada's Broadcasting Policy (Kingston, McGill-Queen's University Press, 1990). 
la radiodiffusion, qui voulaient une radio canadienne distincte de sa voisine américaine, et ceux qui tentèrent (avec un certain succès) d'exploiter son formidable potentiel commercial. S'affrontèrent ainsi deux conceptions, l'une reposant sur l'idéal d'un média voué à l'éducation et à l'unité nationale, et l'autre sur l'utilisation de la radio comme instrument de divertissement et de profit. Telle qu'elle s'est développée, la radiodiffusion canadienne apparaît comme un compromis parfois impossible entre ces deux positions extrêmes représentées, entre autres, par l'Association canadienne des radiodiffuseurs ${ }^{11}$ et par la Ligue canadienne de la radiodiffusion dont le porte-parole et figure de proue du nationalisme canadien, Graham Spry, exhortait les Canadiens à choisir entre «L'État et les États-Unis» (the State or the United States) en $1932^{12}$.

Ces groupes d'intérêt - et bien d'autres - se sont disputé l'espace public par le truchement de commissions, groupes de travail et autres enquêtes gouvernementales dont le comité Juneau constituait l'an dernier le plus récent exemple. Dès 1929, la commission Aird reconnaissait la nécessité pour le gouvernement fédéral d'assurer la gestion et le financement de la radio et de la décharger ainsi de ses obligations commerciales. Bien qu'à des degrés divers, les principes énoncés par cette première enquête publique furent retenus avec la création, en 1932, de la Commission canadienne de la radio qui allait devenir quatre ans plus tard la Société Radio-Canada. Celle-ci imprimait à la radiodiffusion canadienne une structure juridique et politique distincte de son homologue (et concurrente) américaine ${ }^{13}$. Réaction à l'impérialisme économique américain de même qu'expression d'un nationalisme canadien en germe, cette évolution découle également de l'influence de la Grande-Bretagne qui s'était dotée de la British Broadcasting Corporation au cours des années 1920. Le financement de la Canadian Broadcasting Corporation (Société Radio-Canada) fut ainsi à la charge du trésor public. Mais les stations privées échappèrent à la nationalisation annoncée par la loi sur la radiodiffusion, tout en étant frappées de l'interdiction de se constituer en réseaux privés. Cette interdiction fut levée par la refonte de la loi en 1958. Entretemps, la radio publique fut rapidement confrontée aux défis qu'impose le contexte canadien: vaste étendue géographique, régionalismes et faiblesse démographique face aux puissants États-Unis. Par ailleurs, le réseau «national» allait bientôt se scinder en services anglais et

11. T.-J. Allard, L'histoire de l'ACR, 1926-1976: radio et télévision privée au Canada (Ottawa, Association canadienne des radiodiffuseurs, 1976).

12. Canada, Comité spécial de la Chambre des communes sur la radiodiffusion (Ottawa, Imprimeur du Roi, 1932).

13. F. Peers, op. cit. 
français. Dans ces conditions de production d'un contenu canadien suffisant et varié, les subventions ne suffirent pas et le recours aux émissions commanditées, américaines de surcroît, devint inévitable ${ }^{14}$. Leonard W. Brockington, président de la $\mathrm{CBC}$, l'admettait déjà en 1938:

Sincere objections are voiced to the broadcasting of so-called American commercial programmes. Let us face the facts together, you and I, in a commonsense way. We have at present on our networks eight and one-half hours per week of so-called American commercial programmes, out of a total of ninety-eight broadcasting hours. It is admitted that they occupy some particularly attractive radio time. Their entry into Canada is not a new thing. [...] Those programs came into Canada free over American stations. Today, a few, especially selected for their value as entertainment, come into your homes throughout the land, through your Canadian stations. For that privilege they contribute to the funds necessary for the construction and maintenance of your stations and the improvement of your programmes. [...] These programmes are all sponsored by companies engaged in distribution, assembling or manufacture within $\mathrm{Ca}$ nada. The goods advertised are practically all made and sold in Canada, by Canadians. [...] It is true that the programmes originate in the United States. [...] We belong, willy nilly, to the North American continent and North American civilisation ${ }^{15}$.

Depuis le début, en effet, il semble que la diffusion des émissions américaines au Canada ait été dictée par le fort rendement d'une telle opération: les coûts de production sont relativement peu élevés aux États-Unis, où les moyens sont plus substantiels, le marché plus grand et les économies d'échelle possibles. Ainsi, l'argument économique restera longtemps en toile de fond des grands débats sur la radiodiffusion canadienne, la Société Radio-Canada n'ayant pas les moyens d'assumer seule la production d'une grille de programmation entièrement canadienne, en dépit du mandat que lui confiait la loi.

$\mathrm{Au}$ Canada, les normes en matière de publicité furent plus ou moins contraignantes. Dès 1933, la Commission canadienne de la radiodiffusion réglementait la réclame de certains produits pharmaceutiques, imposait un maximum de temps d'antenne à la publicité et

14. A. Weir, op. cit., 225.

15. Société Radio-Canada, Chatting with the Listener (Ottawa, Société Radio-Canada. Transcription d'un discours prononcé sur les ondes du réseau national le 3 février 1938), 5-6. Voir Archives nationales du Canada, RG 41, Documents de la Société Radio-Canada, Vol. 472, Dossier 31-9. 
allait même jusqu'à l'interdire complètement le dimanche ${ }^{16}$. Ces mesures furent néanmoins empreintes de compromis. D'une part, la règle prévoyait que les émissions étrangères ne pouvaient constituer plus de $40 \%$ du temps d'antenne, mais, d'autre part, elle stipulait qu'une émission d'origine étrangère était considérée canadienne moyennant quelques arrangements de nature publicitaire: «A program of foreign origin which advertises goods manufactured in Canada, and names the address in this country where such goods are produced and distributed, shall be deemed a Canadian program ${ }^{17}$.»

En matière de publicité, la loi sur la radiodiffusion de 1936 (dont la refonte ne surviendra qu'en 1958) prescrivait une limite de $10 \%$ de tout programme: sur l'avis de la SRC, une station transgressant cette règle devait immédiatement se conformer aux critères de qualité et de quantité de la publicité. De plus, les annonces éclair (spots publicitaires), réservées aux stations affiliées et privées ${ }^{18}$, ne pouvaient excéder deux minutes par heure de diffusion et demeuraient frappées d'interdiction en soirée les jours de semaine (entre $19 \mathrm{~h} 30$ et $23 \mathrm{~h}$ ) et en tout temps le dimanche. Finalement, afin d'en vérifier la bonne marche, toutes les stations étaient tenues de fournir à Radio-Canada - qui fut à la fois diffuseur national et organisme de contrôle du système canadien de radiodiffusion jusqu'à ce que le Bureau des gouverneurs de la radiodiffusion prenne la relève en 1958 - un registre des programmes contenant, entre autres choses, l'identification des émissions ainsi que la durée et la date de chaque annonce éclair, de même que le nom des commanditaires de toutes les émissions. Les fiches de commandite sur lesquelles se fonde le présent article sont sans doute liées à cette obligation.

La Société Radio-Canada prenait également des mesures pour que la publicité respecte la morale et le bon goût ${ }^{19}$. Une directive interne datée de 1949 précisait que la radio ne pouvait faire référence à la ménopause, à l'hygiène corporelle ou à des produits laxatifs autrement qu'avec finesse; elle allait jusqu'à proscrire les effets sonores imitant les bruits des prothèses mal arrimées dans les annonces de fixatifs à dentiers $^{20}$ ! Elle veillait surtout à limiter la diffusion d'émissions com-

16. Commission canadienne de la radiodiffusion, Rapport annuel, 1935-1936 (Ottawa, Imprimeur du Roi, 1936).

17. Commission canadienne de la radiodiffusion, Rules and Regulations (Ottawa, Imprimeur du Roi, 1933).

18. A. Weir, op. cit., 228-229.

19. Société Radio-Canada, Règlements concernant les stations de radiodiffusion adoptés en vertu de la Loi canadienne sur la radiodiffusion (Ottawa, Radio-Canada, 1941) (règlements de 1936 révisés et modifiés le 24 mars 1941).

20. Canadian Broadcasting Corporation, Regulation No.12: Procedure for Clearance of Food and Drug Radio Continuity (Toronto, Broadcast Regulations Division, 1949), 5-7. 
manditées. Si l'on en juge d'après la déclaration de Leonard Brockington, que nous avons citée plus haut, ces émissions étaient du reste sélectionnées selon de stricts critères de qualité, une démarche dont le sens exact reste difficile à saisir, mais qui renvoie certainement à l'aversion éprouvée par certaines élites à l'endroit des émissions américaines et, plus particulièrement, à l'endroit de la culture populaire. Ce point de vue, qui n'admet pas que la masse des auditeurs cherche avant tout le divertissement, considère le public comme un bloc inerte mais malléable au gré de la direction bienveillante d'une élite éclairée, c'est-à-dire consciente de sa mission. Augustin Frigon, membre de la commission Aird et futur directeur général de la Société Radio-Canada, résume parfaitement cette idée lors d'audiences publiques tenues à Québec le 5 juin 1929: «You talk about jazz. I like jazz, but I like good jazz. As a public body we should see that some means are taken to eliminate poor jazz because it is bad for the mentality of the public. Although you would like to satisfy everybody, certain things are better off the air than on. The well-learned should think and do something about it $^{21}$.» Esthétique, savoir et dignité constituent donc des motifs avancés pour justifier le redressement d'une radio qui en appelle trop aisément à la culture populaire. Peu importe ses préférences, le public est un objet à soumettre aux intérêts supérieurs, que ce soit en termes politiques, moraux ou culturels. Le mémoire présenté par Alexis Gagnon du journal Le Devoir à la commission Aird (1929) est fort éloquent à ce propos: «On doit donner au peuple ce qui sera bon pour lui, quitte à lui accorder ce qui lui plaît davantage quand la chose est possible. L'éducation du peuple au beau et au bien est l'idéal que doit poursuivre la radio [...]. Evidemment il faudra s'y prendre avec tact et discrétion, mais puisque la chose est possible, pourquoi n'utiliserait-on pas la radio pour élever le niveau de culture esthétique et individuelle de notre peuple ${ }^{22}$ ?» $\mathrm{Ce}$ discours n'est d'ailleurs pas unique ni spécifique à la radio. Il a accompagné l'apparition de chacun des médias modernes y compris le cinéma ${ }^{23}$.

Dans ce contexte idéologique, qui est à l'origine de RadioCanada, la radio canadienne est devenue un service public et non pas une industrie. Mais, pour les raisons que nous avons mentionnées, il y eut malgré tout commandite à la radio tant publique que privée, tant

21. Archives nationales du Canada, RG 42 Documents du ministère de la Marine et des Pêcheries, Vol. 1077, Dossier 227-10-8, Audiences de la Commission Aird, 15.

22. Archives nationales du Canada, RG 33/14, Documents de la Commission Aird, Vol. 5, 227-10-6, mémoire $\mathrm{n}^{\circ} 2,6$.

23. R. De la Garde, «Y a-t-il un public dans la salle?» dans M. Beauchamp, dir. Communication publique et société: repères pour la réflexion et l'action (Boucherville, Gaëtan Morin, 1991), 243-283. 
francophone qu'anglophone. Quelle a été l'ampleur de cette publicité et qui en furent les générateurs? La réponse à cette question pourrait rendre compte de la commercialisation de la radio publique et, par ricochet, de l'influence du modèle américain.

La nationalisation de la radio canadienne fut en grande partie justifiée dans le discours officiel (celui du gouvernement fédéral et des élites nationalistes pancanadiennes) par la menace des États-Unis dont l'hégémonie économique semblait déboucher en impérialisme culturel $^{24}$. D'ailleurs, l'incapacité de doter le Canada d'une structure radiophonique parfaitement distincte a certainement accéléré l'injection de produits culturels étrangers dans le milieu canadien: la commercialisation des ondes radio serait ainsi le symptôme d'un matérialisme souvent associé à l'American way of life. Pourtant, davantage qu'une culture authentiquement étatsunienne (si cela existe), la commercialisation de la radio pourrait bien avoir moussé l'émergence d'une culture de masse. Si, au Canada, la publicité d'origine américaine entretient des rapports de cause à effet avec la diffusion d'émissions étrangères, il n'en va pas forcément de même au Québec (ou au Canada) français. Et, ainsi, une influence américaine plus indirecte n'a pas nécessairement contrevenu au développement d'un imaginaire québécois, d'une américanité québécoise, pourrait-on dire. Selon Gérard Bouchard et Yvan Lamonde, l'américanité découlerait des «nouvelles formes culturelles qui se sont mises en place depuis le XVII ${ }^{e}$ siècle à la suite des transferts migratoires de l'Europe vers les Amériques et qui reflètent la somme des ruptures, des processus de différenciation (par invention, adaptation) et des projets de recommencement collectif caractéristiques de plusieurs collectivités neuves ${ }^{25}$ ». En visant le grand public au moyen du divertissement populaire, la publicité radiophonique pourrait bien s'être insérée au cœur de ce processus.

24. M. Filion, Radiodiffusion et société distincte. Des origines de la radio jusqu'à la Révolution tranquille au Québec (Montréal, Éditions du Méridien, 1994); «Broadcasting and Cultural Identity: the Canadian Experience», Media, Culture and Society, 18,3 (1996): 447-468.

25. G. Bouchard et Y. Lamonde, dir., Québécois et Américains: la culture québécoise aux XIXe et XXe siècles (Montréal, Fides, 1995), 8. Voir également G. Bouchard, Entre l'Ancien et le Nouveau monde: le Québec comme population neuve et culture fondatrice (Ottawa, Presses de l'Université d'Ottawa, 1996) et Y. Lamonde, Ni avec eux ni sans eux: le Québec et les ÉtatsUnis (Québec, Nuit Blanche, 1996). En matière de communication de masse, d'autres recherches montrent que des «petites nations» peuvent emprunter à un puissant voisin sans pour autant cesser de se développer une identité propre: voir à cet égard R. De la Garde, W. Gilsdorf et I. Wechselmann, dir., Small Nations, Big Neighbour. Denmark and Québec/Canada Compare Notes on American Popular Culture (Londres, John Libbey, 1993). 


\section{II - LA PUBLICITÉ À LA RADIO DE RADIO-CANADA, 1938-1958}

Le produit de la radio est éphémère et volatile. Ainsi, les sources font souvent défaut non seulement sur la publicité mais sur les émissions elles-mêmes. À notre connaissance, il n'existe aucune collection complète - loin s'en faut - d'enregistrements sonores. Dans le cas qui nous occupe, il faut recourir à d'autres sources afin de déterminer la fréquence de la réclame publicitaire, ses origines et son incidence sur la grille de programmation. Heureusement, nous disposons de tels documents.

Créées à des fins administratives, les fiches de commanditaires d'émissions radiophoniques de la Société Radio-Canada sont conservées aux Archives nationales du Canada ${ }^{26}$. Elles sont réparties en diverses séries dont celle du réseau national qui fut le principal vecteur des grandes campagnes publicitaires, en raison, justement, de son caractère national: «depuis l'établissement du réseau [Radio-Canada], dans les provinces anglaises comme dans le Québec, plusieurs commanditaires ont cessé de faire le gros de leur réclame par les postes privés afin de bénéficier du plus vaste auditoire de Radio-Canada», affirmait à cet égard Paul Legendre en $1947^{27}$. Les quelque 568 fiches qui composent cette série correspondent à autant de contrats de commandite. Pour chacun d'eux, on trouve le nom du commanditaire, habituellement identifié par sa raison sociale bien qu'en de rares occasions il le soit aussi par sa marque de commerce, le titre de l'émission commanditée, les dates et l'horaire de diffusion, le nombre total d'épisodes commandités (jusqu'à 260 par année lorsqu'il s'agit d'une émission quotidienne), ainsi que le nom et l'adresse de l'agence publicitaire responsable de la négociation du contrat. Ces données permettent de saisir l'évolution quantitative de la commandite au cours de la période à l'étude, d'identifier les principaux commanditaires et de saisir leurs orientations stratégiques comme le choix des émissions et, par ricochet, celui du marché visé. Précisons que le réseau français se composait de stations de base, c'est-à-dire appartenant en propre à Radio-Canada (CBF-Montréal, CBV-Québec, CBJ-Chicoutimi et CBAF-Moncton), ainsi qu'un nombre variable de stations affiliées. Depuis les années 1940, le rayonnement du réseau couvrait l'ensemble du Québec habité et ses régions limitrophes.

26. Archives nationales du Canada, RG 41 Documents de la Société Radio-Canada, Acquisition 1986-87/162.

27. P. Legendre, La publicité radiophonique dans la Province de Québec, mémoire de maîtrise, Université Laval, 1947, 56. 


\section{1 - ÉVOLUTION DE LA COMMANDITE}

Afin de mesurer le volume de la publicité et son évolution, nous avons retenu comme indicateur le nombre de fois où une émission était commanditée, ce qui revient en fait au nombre d'épisodes et correspond, par analogie, à la fréquence de parution d'une réclame publicitaire dans un quotidien. Cette unité, que nous nommerons épisode/émission, place toutes les mentions au même niveau d'importance bien que certaines d'entre elles - par exemple celles qui se retrouvent dans le titre même des émissions comme le CourrierConfidence Camay - ont peut-être eu un plus grand impact. Mais cela reste à démontrer et seules quelques émissions présentent cette caractéristique. Par ailleurs, notre unité de mesure (le nombre d'épisodes) privilégie les émissions quotidiennes au détriment des hebdomadaires; les premières (des radiofeuilletons pour la plupart) sont presque toujours découpées en épisodes d'un quart d'heure contre une durée plus longue pour les autres (le hockey par exemple). Sauf quelques rares exceptions, la publicité est confinée à la mention de la firme commanditaire ou du produit à promouvoir au début et, parfois, à la fin de l'émission. La portée réelle de chaque réclame publicitaire peut avoir varié, mais il est généralement admis que le martèlement finit par rejoindre le consommateur ${ }^{28}$. Une étude menée en 1952 pour le compte de la Société Radio-Canada indique justement que la principale source d'insatisfaction de l'auditoire était alors la quantité d'annonces publicitaires ${ }^{29}$.

Pour les fins de notre exercice, nous avons calculé le nombre total d'épisodes/émissions commandités annuellement sur les ondes du réseau français. Première constatation, le volume de la commandite croît substantiellement et presque régulièrement jusqu'en 1954, un résultat qu'il faut mettre en parallèle avec l'accroissement de la programmation et la ferveur populaire dont jouit la radio ${ }^{30}$.

28. P. Rutherford, «The Culture of Advertising», Canadian Journal of Communication, 13,3-4 (1983): 102-113.

29. Gruneau Research Limited, Survey of Canadian Public Opinion Towards Radio and the Canadian Broadcasting Corporation (Toronto, Gruneau Research Limited, 1952).

30. La radio québécoise s'est développée en synchronie avec le reste du monde occidental depuis la fondation de la première station commerciale à Montréal en 1919, XWA, qui deviendra bientôt CFCF (voir P. Pagé, «Montréal et la naissance d'une nouvelle culture publique: la 'Semaine de la radio' dans le journal La Presse (1924-1934)», Fréquence/Frequency, 1-2 (1995): 165-192). À la suite de l'émergence de nombreuses autres stations, telle CKAC en 1922, l'usage de la radio s'est répandu. Si bien qu'à la veille de l'apparition de la télévision (en 1952), à qui elle a très certainement ouvert la voie de la ferveur populaire, la radio était parvenue à investir la très grande majorité des foyers québécois et canadiens (voir E. Lavoie, «L'évolution de la radio au Canada français avant 1940», Recherches sociographiques, 12,1 (1971): 17-49). 


\section{TABLEAU 1}

\section{NOMBRE D'ÉPISODES/ÉMISSIONS COMMANDITÉS à La RADIO FRANÇAISE DE RADIO-CANADA, 1938-1958}

$\begin{array}{rrrr}1938 & 299 & 1949 & 4238 \\ 1939 & 812 & 1950 & 4029 \\ 1940 & 2188 & 1951 & 4346 \\ 1941 & 2658 & 1952 & 4349 \\ 1942 & 3031 & 1953 & 4442 \\ 1943 & 2963 & 1954 & 4780 \\ 1944 & 3142 & 1955 & 4181 \\ 1945 & 3042 & 1956 & 3726 \\ 1946 & 3127 & 1957 & 2260 \\ 1947 & 2805 & 1958 & 581 \\ 1948 & 3680 & \text { Total } & 64888\end{array}$

Source: Archives nationales du Canada, RG 41, Documents de la Société Radio-Canada, Acquisition $1986-87 / 162$.

Mais les recettes publicitaires chutent considérablement à partir de 1952-1953 jusqu'à la fin de la décennie. Le nombre record de 27 commanditaires en 1949 tombe à 13 en 1959. Cette désaffection s'explique peut-être par la croissance de la radio privée ${ }^{31}$, mais aussi par l'arrivée de la télévision qui draine rapidement les ressources: de 4157000 dollars en 1954-1955, ses recettes publicitaires grimpent à 30127000 dollars en 1958-1959, alors que celles de la radio ne cessent de baisser (tableau 2). À ce transfert s'ajoutent d'autres éléments d'explication comme le fait que la société d'État ait accepté de se conformer à la recommandation émise par la Commission royale d'enquête sur l'avancement des arts, lettres et sciences au Canada (commission Massey) en 1951 de refuser toute réclame commerciale locale pour les postes qu'elle exploitait directement ${ }^{32}$. Cette politique fut renversée en 1959 à la suite des recommandations de la Commission royale d'enquête sur la radio et la télévision (commission Fowler) de 1957.

31. O. J. Firestone, op. cit., 130.

32. Société Radio-Canada, La radio commerciale de Radio-Canada: étude du service de la politique commerciale et du service des ventes (Ottawa, Société Radio-Canada, 1970), 3. 
TABLEAU 2

ReCeTtes PUBlicitaires NETTES DE LA RADIO

de Radio-Canada, 1938-1960

(EN MILLIERS DE DOLLARS)

$\begin{array}{rrrr}1938-1939 & 663 & 1949-1950 & 2695 \\ 1939-1940 & 794 & 1950-1951 & 2803 \\ 1940-1941 & 1026 & 1951-1952 & 2803 \\ 1941-1942 & 1169 & 1952-1953 & 2903 \\ 1942-1943 & 1327 & 1953-1954 & 2853 \\ 1943-1944 & 1533 & 1954-1955 & 2468 \\ 1944-1945 & 1793 & 1955-1956 & 2119 \\ 1945-1946 & 1835 & 1956-1957 & 1564 \\ 1946-1947 & 1998 & 1957-1958 & 1661 \\ 1947-1948 & 2086 & 1958-1959 & 1372 \\ 1948-1949 & 2493 & 1959-1960 & 1489\end{array}$

Source: Société Radio-Canada, La radio commerciale au Canada: passé, présent, potentiel (Ottawa, Société Radio-Canada, Service de la politique commerciale et du programme des ventes, 1970), 5 .

\section{2 - LES COMMANDITAIRES}

Des sommes considérables sont investies dans la publicité radiophonique de Radio-Canada, en particulier au cours de ce que d'aucuns ont appelé l' «âge d'or» de la radio. D'où provenaient-elles? «Actuellement, la majorité de nos programmes commandités le sont par des firmes étrangères», affirmait Paul Legendre en $1951^{33}$. Nos sources lui donnent raison puisque la plupart des quelque 75 commanditaires dont nous avons relevé la présence sont américains. En effet, les entreprises canadiennes, comme Molson et Canada Packers, viennent en fin de liste si l'on en juge d'après le nombre d'épisodes/émissions commandités.

33. Paul Legendre, La radio, puissance sociale (Québec, Institut littéraire du Québec, 1951), 81. 


\section{TABLEAU 3}

PrincipauX CommanditaIRES deS Émissions RADIOPHONIQUeS DIFFUSÉES AU RÉSEAU FRANÇAIS DE RADIO-CANADA 1938-1958

Commanditaire

Nombre total d'épisodes/émissions commandités

Procter \& Gamble

Lever Brothers

Campbell Soup

Colgate Palmolive

Sterling Drugs

General Foods

Robin Hood Flour Mills

Carnation Milk

S. C. Johnson

Javex

Total
20137

14751

4020

3584

2614

2176

1483

1455

943

772

51935

Source: voir tableau 1.

Le groupe de dix firmes mentionnées dans le tableau 3 génère à lui seul $80 \%$ des épisodes/émissions commandités dont témoignent nos fiches: $31 \%$ pour Procter \& Gamble et $22,7 \%$ pour sa concurrente Lever Brothers, bonne deuxième. L'année 1947 constitue un record de concentration alors que ces fabricants commanditent à eux deux $68 \%$ des épisodes/émissions au réseau français. Puisque leurs produits (les savons Sunlight, Lux, Ivory et Camay, ainsi que les lessives Chipso, Rinso et Oxydol) occupaient dans le marché le même créneau, il est facile d'imaginer l'âpre rivalité qui a pu animer la commandite des «romans-savons» dont l'appellation prend ici tout son sens.

Signalons que le gouvernement fédéral s'est aussi intéressé à la réclame, dans une bien moindre mesure cependant. Pendant la Seconde Guerre mondiale, le ministère des Finances parrainait l'émission L'heure de la victoire, notamment pour promouvoir l'achat des bons... de la victoire. De même, le ministère de la Défense nationale a utilisé la radio pour encourager le recrutement jusqu'à la guerre de 
Corée, en commanditant des émissions comme Frères d'équipage (1942), En plein vol (1943), Tambour battant (1949-1954), Coup de clairon (1950), Béni fut son berceau (1951), La chanson de l'escadrille (1952-1953) et La chanson du CARC (1954). Au total, nous avons relevé 1691 épisodes/émissions commandités par des agences gouvernementales, particulièrement la Commission des prix et du commerce en temps de guerre et le Comité national des finances de guerre. La démarcation entre la publicité (marketing social) et la propagande pourrait s'avérer ici bien fragile. Renée Legris a analysé le contenu de quelques radiofeuilletons, tels Rue principale et Béni fut son berceau, diffusés à $\mathrm{CBF}$, tête du réseau français: tant en ce qui a trait à la commandite de ces émissions qu'à leur trame même, Legris affirme qu'elles ont constitué des moyens de propagande à l'endroit du Québec réfractaire à la participation du Canada à la guerre ${ }^{34}$.

\section{3 - LES ÉMISSIONS COMMANDITÉES}

En recommandant que la publicité soit progressivement éliminée à l'antenne de Radio-Canada, le rapport Juneau (1996) établit un lien inévitable entre la réclame, c'est-à-dire la commercialisation, et le divertissement populaire. Auparavant, le rapport de la commission Aird (1929), celui de la commission Massey (1951) et bien d'autres encore ont également déploré les liens entre la culture de masse et la consommation de masse, à plus forte raison lorsque toutes deux étaient associées à l'américanisation. Faut-il rappeler que l'instauration de la radio publique au Canada visait à satisfaire les aspirations d'une élite nationaliste, particulièrement active au Canada anglais ${ }^{35}$.

Bien que le lien entre la réclame publicitaire et la «popularité» des programmes ne soit pas absolu (la commandite du Metropolitan Opera importé du réseau américain NBC le samedi après-midi par la compagnie McColl-Frontenac Oil constitue une bonne exception), il ressort que les commanditaires visaient généralement à rejoindre la masse des auditeurs: «[...] les produits annoncés à la radio sont, en majorité, ceux que la masse du peuple consomme régulièrement. C'est donc au peuple que s'adresse le programme commandité», affirmaient à ce sujet Girardet et Legendre ${ }^{36}$, ce qui n'est pas pour surprendre. Le tableau 4 énumère la dizaine d'émissions apparemment les plus prisées par les commanditaires au cours de la période à l'étude.

34. Renée Legris, Propagande de guerre et nationalisme dans le radio-feuilleton, 1939 1955 (Montréal, Fides, 1981).

35. M. Filion, «L'américanisation de la radio québécoise et l'émergence du service national de radiodiffusion au Canada», Communication, 14,2 (1993): 197- 221.

36. P. Girardet et P. Legendre, op. cit., 169. 


\section{TABLEAU 4}

Émissions du RéSEau français de Radio-Canada LES PLUS FRÉQUEMMENT COMMANDITÉES 1938-1958

Émissions

Quelles nouvelles?

Rue principale

Les joyeux troubadours

Jeunesse dorée

Un homme et son péché

Métropole

Grande sour

Francine Louvain

Maman Jeanne

Tante Lucie

Total
Principaux

commanditaires

Procter \& Gamble

Procter \& Gamble

Colgate Palmolive

Quaker Oats

Tea Council of Canada

McCormick Biscuits

Campbell Soup

Fry Cadbury

Success Wax

Lever Brothers

Campbell Soup

Procter \& Gamble

Lever Brothers

Procter \& Gamble

Lever Brothers

Sterling Drugs

Lever Brothers
Nombre

d'épisodes/émissions
4994

4961

4801

4256

3188

3121

2994

2940

2429

2075

35259

Source: voir tableau 1.

Pas de surprise: ces dix émissions renvoient toutes à ce que l'on classe généralement dans la catégorie du «divertissement léger», c'està-dire des radiofeuilletons et des programmes de variété qui touchent de vastes auditoires. En 1952, la firme de sondage Gruneau relevait des cotes d'écoute exceptionnelles pour Rue principale $(73 \%$ de l'auditoire total), Jeunesse dorée (72\%), Maman Jeanne (72\%) et Grande sæur $(71 \%)^{37}$. Certaines des émissions radiophoniques les plus populaires franchiront d'ailleurs allègrement le pas vers la télévision et connaîtront encore au petit écran de grands succès: la palme revient à ce sujet aux Belles histoires des pays d'en haut, une adaptation du livre Un homme et son péché de Claude-Henri Grignon.

37. Gruneau Research Limited, A Study of Radio Listenership in the Province of Quebec (Toronto, Gruneau Research Limited, 1952). 
Fait important à signaler, parmi les émissions que nous avons relevées en faisant le décompte de la publicité, il en est très peu qui soient d'origine américaine, sinon le Metropolitan Opera, au demeurant fort éloigné du divertissement populaire. Il apparaît clairement que la commandite d'outre-frontière, malgré son poids, n'entraine pas avec elle une programmation étrangère au réseau français de RadioCanada.

Ce qui ne veut pas dire que la culture canadienne-française n'a pas été altérée pour autant par la radio et sa programmation: à cet égard, Elzéar Lavoie ${ }^{38}$ a insisté sur la normalisation de la langue et sur l'exclusion, par la SRC, d'une commandite en appelant à des valeurs ou à des expressions jugées «trop» populaires. Paul Legendre parle du rejet de certaines réclames mal traduites ${ }^{39}$, tandis que Jean-Marie Allard affirme que «la sévérité du code [règles concernant la qualité linguistique des annonces imposées dès 1945] fournissait en outre un argument supplémentaire pour convaincre les annonceurs nationaux que les francophones méritaient beaucoup mieux que la traduction de messages conçus en anglais ${ }^{40} \gg$. Par ailleurs, on ne saurait prétendre que les commanditaires n'aient exercé aucune influence sur le contenu des émissions: en plus des classiques Théâtre Ford, Théâtre lyrique Molson et Roman lyrique Victor (pour RCA Victor), on trouve des titres aussi évocateurs que Dans ma tasse de thé commandité par le thé Tenderleaf, Les meuniers mélomanes et Les aventures de Robin des bois parrainés par les producteurs de farine Robin Hood, Les alouettes Eveready par la Canadian National Carbon, La petite cigarettière par L. O. Grothe (distributeur de tabac), La soirée au vieux moulin par Purity Flour Mills, L'invitation à la valse par Slater Shoe, Perrette et ses fredons par la Fédération canadienne des producteurs de lait et La chanson 57 par la Compagnie Heinz du Canada. Une analyse plus poussée de ces émissions - si elles étaient disponibles - permettrait peut-être d'aborder l'importante question de l'influence de la commandite sur le contenu. Bon nombre de ces émissions furent non seulement parrainées, mais également produites par l'annonceur ou son agence ${ }^{41}$. On sait que la firme Radio Programme Producers de Paul Langlais fut très active depuis la conception (écriture des textes, recrutement des artistes, distribution des rôles, etc.)

38. E. Lavoie, «La constitution d'une modernité culturelle populaire dans les médias au Québec, 1895-1950» dans Y. Lamonde et $\mathrm{E}$. Trépanier, dir., L'avènement de la modernité culturelle au Québec (Québec, Institut québécois de recherche sur la culture, 1986), 253-298.

39. P. Legendre, op. cit., 138.

40. J.-M. Allard, op. cit., 49.

41. Ibid., 44. 
jusqu'à la mise en ondes des émissions, en passant par leur vente à des commanditaires ${ }^{42}$.

Ainsi, sauf quelques rares exceptions comme Grande sæur qui était une adaptation par Louis Morisset de Big Sister, la plupart des émissions du réseau français furent crées par des auteurs de renom en matière de littérature radiophonique québécoise: Jovette Bernier (Quelles nouvelles?), Édouard Baudry, René Boivin et Paul Gury (Rue principale), Jean Desprez (Jeunesse dorée), Claude-Henri Grignon (Un homme et son péché), Robert Choquette (Métropole) et Jacques Rudel-Tessier (Maman Jeanne) pour ne citer que ces exemples ${ }^{43}$. Une analyse effectuée dans les fiches de commanditaires de la radio (anglaise) de la Canadian Broadcasting Corporation révèle un tout autre phénomène: comme au Canada français, les firmes américaines sont très présentes, mais elles commanditent en grande partie des émissions directement importées des grands réseaux américains: Amos'n Andy (Lever Brothers - NBC), Ma Perkins (Procter \& Gamble - CBS), Lux Radio Theatre (Lever Brothers - CBS), Big Sister (Procter \& Gamble - CBS) et Kraft Music Hall (Kraft - NBC) pour ne citer qu'elles. Dans ce cas, la publicité peut être étroitement et directement associée au divertissement populaire d'origine américaine.

Au Québec, la publicité n'en est pas moins génératrice de divertissement léger, une situation qui n'eût pas l'heur de plaire aux élites. En déplorant l'importance prise par les radioromans et les émissions de variété, la commission Massey (1949-1951) se donne la mission de veiller à l'éducation du public comme l'avaient fait auparavant la commission Aird et la Ligue canadienne de la radiodiffusion lors du débat qui mena à la nationalisation de la radio en 1932. Selon le rapport Massey, la constitution d'une identité canadienne passe nécessairement par l'amélioration générale du niveau de culture. Citant d'entrée de jeu saint Augustin selon qui la qualité d'un peuple réside en ce qu'il aime, le rapport présente une vision édulcorée d'un passé encore à l'abri de la spoliation des temps modernes: «Le goût musical d'une bonne partie de la population était formé, dans une grande mesure, par des musiciens bien exercés, qui apportaient chez nous toute une tradition de belle musique ${ }^{44} . \gg$ Ce qui constitue la belle musique, Massey ne le dit pas; cependant, le rapport réagit fortement à la popularité de certains genres tels les feuilletons radiophoniques

42. P. Girardet et P. Legendre, op. cit., 172; A. Weir, op. cit., 312; H. E. Stephenson et C. McNaught, op. cit., 297.

43. P. Pagé et al., Répertoire des auvres de la littérature radiophonique québécoise (Montréal, Fides, 1975).

44. V. Massey, Rapport de la Commission royale d'enquête sur l'avancement des arts, lettres et sciences au Canada (Ottawa, Imprimeur de la Reine, 1951), 23. 
(au réseau français) dont la plupart verseraient «dans le mélodrame, l'irréel, l'abus de lieux communs et des clichés» et s'inscriraient ainsi en rupture avec la culture traditionnelle ${ }^{45}$. Nul doute que les commissaires furent sensibles au mémoire déposé par Pierre Boucher qui déplorait l'inculture des radioromans ${ }^{46}$. C'est donc tout autant, sinon plus, le genre que l'origine des émissions qui inquiète les commissaires lorsqu'ils déclarent mettre en doute la possibilité «de concilier les buts déclarés de la radio nationale et la proportion beaucoup trop considérable de romans-feuilletons qu'elle transmet ${ }^{47}{ }^{»}$.

\section{4 - MARCHÉ VISÉ PAR LA PUBLICITÉ}

La grande majorité des émissions commanditées renvoient au divertissement léger: des radiofeuilletons pour la plupart, mais aussi des programmes de variété qui sont regroupés dans des créneaux horaires privilégiés. On ne trouve pas moins de $61,6 \%$ des épisodes/émissions commandités entre $10 \mathrm{~h}$ et $14 \mathrm{~h}$, mais seulement $21,5 \%$ après $18 \mathrm{~h}$. En fait, c'est le jour, entre $8 \mathrm{~h}$ et $18 \mathrm{~h}$, qu'on trouve la très grande majorité des épisodes/émissions commandités: $80 \%$ de 1938 à 1945, 75,4\% de 1946 à 1953 et 86,9\% de 1954 à 1958 . Le déplacement de l'écoute en soirée vers la télévision explique probablement l'accroissement proportionnel qui se manifeste dans la commandite de jour au cours de la dernière période.

Par ailleurs, certains types de produits annoncés occupent clairement le plus gros de la commandite. Regroupés en une même catégorie, les produits d'alimentation, de soins de santé, d'hygiène corporelle et d'entretien ménager touchent $87,6 \%$ de l'ensemble des réclames diffusées de 1938 à $1945,90,4 \%$ de 1946 à 1953 et 94,5\% de 1954 à 1958.

Il se dégage de ces chiffres que l'auditoire de jour a constitué la principale cible du diffuseur et de ses commanditaires. Le classement des émissions les plus fréquemment commanditées (tableau 4) en rend compte: parmi elles, seules Métropole et Un homme et son péché sont diffusées en soirée. À une époque où la plupart des femmes étaient au foyer pour ne pas encore avoir investi le marché du travail ou pour l'avoir quitté à la fin de la Seconde Guerre mondiale, on peut avancer qu'elles constituaient certainement le principal marché visé par la commandite, ce qui explique en grande partie la diffusion diurne des radiofeuilletons et des émissions de variété. Ainsi, il n'est pas illogi-

45. Ibid., 43.

46. Archives nationales du Canada, RG 33/28, Documents de la Commission Massey, Vol. 53, Mémoire de Pierre Boucher: La valeur culturelle des radioromans (1950).

47. V. Massey, op. cit., 47. 
que que l'émission Entre nous Mesdames ait été commanditée par les fabricants des savons Lux, Rinso et Sunlight, et que Vie de femmes l'ait été par Javex. Nous nous demandons si la publicité destinée à un auditoire masculin (comme la bière Molson annoncée lors des matchs de hockey) ne s'est pas exprimée par d'autres moyens. Mais, à cet égard, l'étude de Côté et Daigle laisse entendre que la clientèle féminine fut également visée dans les quotidiens québécois qui proposaient aussi une grande part de réclames liées à l'entretien ménager ${ }^{48}$. La commandite radiophonique a certainement joué dans la construction de la grille-horaire, car il semble que les projets du département de la programmation furent souvent contrariés par la préséance attribuée aux émissions commanditées ${ }^{49}$.

\section{5 - UNE PUBLICITÉ AMÉRICANISÉE OU AMÉRICANISANTE?}

D'après nos indicateurs, la publicité radiophonique au réseau français de Radio-Canada entre 1938 et 1958 touche presque exclusivement des émissions de divertissement léger diffusées surtout le jour à l'intention d'un auditoire féminin. Elles sont commanditées très massivement par des fabricants américains de produits courants de consommation. Cette publicité eut-elle une influence directe sur l'imaginaire québécois, comme le craignait Paul Legendre lorsqu'il déclarait en 1951 que «les textes publicitaires dialogués, rimés ou chantés qui accompagnent nos programmes ne sont donc le plus souvent que des traductions ou des adaptations françaises d'annonces étrangères. Nos propres formules de réclames, qui s'efforcent souvent de copier les formules américaines, portent rarement l'empreinte de notre personnalité française ${ }^{50} \gg$ ? Nos données révèlent, en effet, que la plupart des campagnes publicitaires sont préparées non seulement par des entreprises étrangères, mais aussi par des agences situées à l'extérieur du Québec.

L'importance numérique des agences américaines s'explique aisément par l'origine des fabricants tels Proctor \& Gamble et Lever Brothers, dont nous avons vu le rôle, qui transigent avec des compatriotes. Non seulement les agences montréalaises sont minoritaires, mais encore s'agit-il souvent de succursales de grandes firmes anglosaxonnes ${ }^{51}$ : «Française d'expression, [la publicité radiophonique qué-

48. L. Côté et J.-G. Daigle, «Publicité de masse et masse publicitaire dans la presse quotidienne au Québec (1929-1957)» dans P. Lanthier et G. Rousseau, dir., La culture inventée. Les stratégies culturelles aux $19^{e}$ et $20^{\circ}$ siècles (Québec, Institut québécois de recherche sur la culture, 1992), 247-265.

49. A. Weir, op. cit., 133.

50. P. Legendre, op. cit., 81.

51. F. Elkin, op. cit. 
bécoise] demeure anglo-saxonne de conception», affirmaient Girardet et Legendre en expliquant cette situation de fait par l'implantation des filiales américaines en Ontario et par la relative rareté de produits finis fabriqués au Québec ${ }^{52}$.

TABLEAU 5

LIEUX D'ORIGINE DES AGENCES DE PUBLICITÉ RESPONSABLES DE LA COMMANDITE DES ÉMISSIONS RADIOPHONIQUES DU RÉSEAU FRANÇAIS de Radio-Canada, 1938-1958

Lieu Épisodes/émissions commandités en $\%$

New York 37,4

Toronto 35,9

Montréal 22,2

Chicago

Source: voir tableau 1.

Les firmes américaines - souvent par filiales canadiennes interposées - ont peut-être saisi plus vite, et avec plus d'intensité, la portée d'une campagne de publicité soutenue et dynamique. À l'instar de Frederick Elkin, on peut toutefois penser qu'elles ont mis un certain temps à reconnaître le caractère particulier du marché canadienfrançais:

In preparing ads for the French Canadians, the standard policy from the very beginning was translation which assumed similar cultures except for language. [...] Besides being expensive, the possibility that French Canadians, as consumers, might also be significantly different in relevant consumption patterns or that they might react against the existing system did not really occur to the English Canadians ${ }^{53}$.

Pourtant, on remarquait dès 1947 l'inefficacité de campagnes publicitaires calquées sur le modèle américain ${ }^{54}$. Ce n'est qu'à partir

52. P. Girardet et P. Legendre, op. cit., 159.

53. F. Elkin, op. cit., 28.

54. P. Legendre, op. cit., 16. 
de 1958 que les publicitaires francophones, jusque-là isolés dans des firmes anglo-saxonnes, parviendront à se regrouper au sein du Publicité-club, cet organisme sans but lucratif devenu l'association des professionnels francophones qui contribuera à l'émancipation du Québec en publicité ${ }^{55}$. Ainsi, bien que difficilement saisissable, la question de l'inefficacité d'une publicité inspirée de l'étranger trouve peut-être sa réponse dans le changement qui s'amorce à la fin des années 1950, alors que des entreprises, particulièrement les brasseries, optent pour des campagnes publicitaires qui puisent dans l'univers spécifique des consommateurs québécois ${ }^{56}$ : du slogan fondé sur la typologie individuelle («Lui, y connaît ça!») incarné par Olivier Guimond à celui qui en appelle à l'identification collective ( $« O n$ est six millions, faut s'parler»), on trouve un éventail de référents au milieu distinct.

\section{CONCLUSION}

La constitution d'une radio «nationale» dans les années 1930 avait pour but avoué de contrecarrer la commercialisation des ondes et de mousser le développement d'une identité culturelle canadienne. Mais, nécessité économique obligeant, la publicité n'en fut pas exclue. Du coup, la radio canadienne publique allait prêter le flanc à l'influence américaine. Au Canada anglais, la pénétration des émissions et de la publicité américaines ne rencontrèrent qu'une faible résistance: quoique tonitruant, le discours d'une élite qui sentait ses valeurs menacées par l'américanisation des ondes ne semble pas avoir eu de grands effets. En matière d'américanisation, la politique canadienne était peut-être adaptée à la situation de la radio de langue anglaise, mais pas nécessairement à celle de la radio de langue française.

En dépit de la publicité, la radio française de Radio-Canada ne put emprunter directement aux producteurs américains: «Les émissions en provenance des États-Unis sont rares au réseau français, ce qui se comprend facilement étant donné la différence de langue. Elles consistaient en deux concerts et en deux émissions de théâtre», soulignait à cet égard Charles Siepmann dans une étude menée pour le compte de la commisssion Massey ${ }^{57}$. Certes, les émissions populaires - celles qui sont commanditées - peuvent avoir été faites à l'américaine, mais il n'en reste pas moins qu'elles ont été écrites, interprétées et produites au Québec pour le grand public francophone qui s'est re-

55. J.-M. Allard, op. cit., 53.

56. Ibid., 97-102.

57. Charles Siepmann, «Aspects de la radiodiffusion au Canada» dans V. Massey, Rapport de la Commission royale pour l'avancement des arts, des lettres et des sciences au Canada (Ottawa, Imprimeur du Roi, 1951), 511-553. 
connu en elles au point d'en faire des succès désormais inscrits dans le patrimoine collectif. «Quand il s'agit de dialogues, de drames ou de comédies, il est nécessaire qu'on les interprète en français et que les textes soient d'esprit québécois. Les commanditaires ont, en général, bien compris la chose et confient à des artistes de chez nous le soin d'écrire, de présenter et de jouer les pièces», affirmait Paul Legendre qui fut non seulement un témoin privilégié de l'époque que nous étudions, mais également un acteur de premier plan en sa qualité de réalisateur de l'émission Chez Miville ${ }^{58}$.

En anglais, le contenu vient avec la publicité, tandis qu'en français la publicité accompagne un contenu québécois. Le milieu de réception, c'est-à-dire le public, dispose au Québec de caractéristiques culturelles propres à l'isoler. La barrière linguistique n'en est pas la moindre. Mais parvint-elle à contrer l'américanisation? Cette notion demeure difficile à mesurer. Nous disposons d'un indice: la présence massive des fabricants américains qui en viennent presque à monopoliser la publicité à la radio de Radio-Canada. Nous ne saurions dire, à partir des sources utilisées dans cette recherche, si cette publicité eut une influence directe et immédiate sur l'évolution des mentalités au Québec entre 1938 et 1958. Notre analyse démontre néanmoins qu'il exista des liens étroits entre la publicité et la promotion des émissions de divertissement léger, fût-ce à la radio publique de Radio-Canada pourtant chargée d'un rôle éducatif. En ce sens, l'influence de la publicité américaine n'en fut pas moins grande pour être indirecte. Mais, au contraire de ce qui s'est produit au Canada anglais, la publicité - bien qu'américaine - a certainement ancré davantage la radio française de Radio-Canada dans son milieu en stimulant la production locale d'émissions de divertissement populaire. Gérard Bouchard affirme que la culture québécoise apparaît tissée par le haut par les élites (qui nient l'américanité) et métissée par le bas par le peuple ${ }^{59}$. Le cas du réseau français de Radio-Canada montre que de 1938 à 1958, et sans doute plus tard aussi, la publicité radiophonique fut un puissant moteur de ce processus de métissage.

58. P. Legendre, op. cit., 71.

59. G. Bouchard, op. cit., 47. 